Research Article

\title{
Doxorubicin and ABT-199 Coencapsulated Nanocarriers for Targeted Delivery and Synergistic Treatment against Hepatocellular Carcinoma
}

\author{
Yun Zhou, ${ }^{1,2} \mathrm{Ke} \mathrm{Li}^{2,3}{ }^{2,}$ an Li, ${ }^{4}$ Shuang Han, ${ }^{5}$ Yang Wang, ${ }^{2,3}$ Xueping Li $\mathbb{D},{ }^{1}$ \\ and Yonghua Zhan $\mathbb{1}^{6}$ \\ ${ }^{1}$ College of Clinical Medicine, Xi'an Medical University, Xi'an, 710021 Shaanxi, China \\ ${ }^{2}$ Shaanxi Key Laboratory of Ischemic Cardiovascular Disease, Shaanxi Key Laboratory of Brain Disorders, Institute of Basic and \\ Translational Medicine, Xi'an Medical University, Xi'an, 710021 Shaanxi, China \\ ${ }^{3}$ Department of Basic Medical Science, Xi'an Medical University, Xi'an, 710021 Shaanxi, China \\ ${ }^{4}$ Hospital of Northwestern Polytechnical University, Xi'an, 710027 Shaanxi, China \\ ${ }^{5}$ The First Affiliated Hospital of Air Force Medical University, Xi'an, 710032 Shaanxi, China \\ ${ }^{6}$ Engineering Research Center of Molecular and Neuro Imaging of the Ministry of Education, School of Life Science and Technology, \\ Xidian University, Xi'an, 710071 Shaanxi, China
}

Correspondence should be addressed to Xueping Li; lxp86@xiyi.edu.cn and Yonghua Zhan; yhzhan@xidian.edu.cn

Received 15 January 2019; Accepted 8 May 2019; Published 8 July 2019

Academic Editor: Surinder Singh

Copyright (C) 2019 Yun Zhou et al. This is an open access article distributed under the Creative Commons Attribution License, which permits unrestricted use, distribution, and reproduction in any medium, provided the original work is properly cited.

\begin{abstract}
For the patients with hepatocellular carcinoma (HCC), conventional chemotherapy is insufficient or has no benefit. Although combination chemotherapy has been proven as an efficient strategy to enhance anti-HCC efficacy, some barriers, such as low bioavailability and side effects, are limiting clinical development. In order to overcome disadvantages of combination chemotherapy in HCC, targeted nanoparticles (NPs) simultaneously loaded with doxorubicin (DOX) and ABT-199 in an optimal synergistic ratio were developed. First, the most synergistic combination with DOX was screened from ABT-199, ABT-263, and ABT-737. Among them, ABT-199 showed optimal synergy with DOX in a ratio of 10:1. Then, cationic amphipathic starch (CSaSt) and hyaluronic acid (HA) were used in coencapsulations of those two drugs. Dual-drug synergistic nanoparticles (DDS NPs) were constructed by absorption of DOX NPs around ABT-199 micelles with an optimal ratio via electrostatic interaction. The shape of DDS NPs was similar to a raspberry, and the size was $112.6 \pm 13.4 \mathrm{~nm}$. The encapsulation efficiencies of DOX and ABT-199 in DDS NPs were $90.2 \pm 4.3 \%$ and $94.7 \pm 2.8 \%$, respectively; meanwhile, the drug loadings were $1.5 \pm 0.4 \%$ and $14.1 \pm 1.1 \%$, respectively. After $72 \mathrm{~h}$ of dialysis, $95 \%$ of ABT-199 remained and less than $50 \%$ of DOX was released. In vitro investigation showed that the drugs in DDS NPs maintained the treated effect in three HCC cell lines; moreover, DDS NPs could perform intracellular delivery of dual drugs and exhibited continuous release of the drugs into different targets. Low in vivo toxicity was found after the acute toxicity test. In vivo fluorescent imaging revealed that DDS NPs could efficiently target and accumulate in the tumor tissues and be maintained more than $72 \mathrm{~h}$ after intravenous injection. Compared with free drugs, DDS NPs with the same dosages exhibited a more significant antitumor effect in the HCC xenograft mouse model. The results indicated that DDS NPs have great potential in HCC chemotherapy.
\end{abstract}

\section{Introduction}

Hepatocellular carcinoma (HCC) is the most primary liver cancer and the second most common cause of cancer- related deaths in the world [1]. The prognosis of HCC is very poor, with only $12.7 \%$ of a 3 -year survival rate and 9 months of a median survival [2]. The increase of HCC and HCC-related death incidence has occurred in the past 
decades in South and East Asia, sub-Saharan Africa, Southern Europe, and North America [3]. HCC could be curatively treated with surgery and liver transplantation in the early stage of malignancy. However, there are only $15 \%$ of patients who are eligible for the further curative treatment, while the other majority of cases will present with serious disease [4]. Chemotherapy is the main clinical treatment in various stages of HCC. Sorafenib is one of the FDAapproved chemotherapeutic medicines for intermediate and terminal HCC. However, sorafenib still does not provide satisfactory effectiveness [5, 6]. Prior to sorafenib, DOX was the most common first-line drug in treatment of advanced HCC. It demonstrated objective response rates ranging from $0 \%$ to $79 \%$ in an initial phase II study [7]. However, severe side effects such as cardiotoxicity and neutropenia occurred due to poor targeting efficacy of DOX, which has caused very serious complications in clinical cancer treatment [8]. Furthermore, chemotherapy-resistant HCC has been reported in many studies [9-11]. Currently, combinational chemotherapy has increasingly attracted the attention in clinical tumor treatment [12]. In contrast with single-drug treatment, combined therapy could impact different pathways in tumor proliferation, metastasis and invasion, thus increasing the chemotherapeutic efficacy and decreasing side effect and tumor resistance $[13,14]$. Several combination treatments were reported in HCC to enhance the antitumor effect. DOX combined with cisplatin caused marginal clinical benefits which included 18.9 of objective response rates, 7.3 months of median overall survival, and median progression-free survival of 6.6 months with tolerable side effect. The cisplatin/interferon alpha-2b/doxorubicin/fluorouracil (PIAF) is a chemotherapeutic combination for treatment of HCC. It is an effective option for HCC patients who have normal liver function. Nevertheless, systemic side effects of PIAF treatment are still inevitable [15-18]. In general, treatment studies of HCC are still not satisfactory, let alone meet the demand of clinical therapy for HCC. Thus, more effective combination chemotherapy is still worthwhile.

The novel development of target therapies focuses on tumor-specific factors or kinases. B cell lymphoma- (Bcl-) 2 family proteins, which are overexpressed in various tumors including HCC, play a very important role in tumor development and treatment resistance [19-21]. Bcl-2 protein can be bound with many proapoptotic factors, such as Bad and Bak proteins, thus inhibiting the process of apoptosis. Therefore, targeting the antiapoptotic $\mathrm{Bcl}-2$ proteins with small molecule compounds will be a promising therapeutic strategy $[22,23]$. Apogossypolone is a Bcl-2 protein inhibitor, and it has demonstrated some benefits in anti-HCC treatment when combined with DOX [24]. Oltersdorf and coworkers discovered ABT-737, which is a small molecule compound used to effectively inhibit Bcl-2 family proteins. The inhibitor exhibited markedly antitumor activity and reduced mortality in a xenograft animal model. The mechanistic research indicates that ABT-737 does not directly induce the apoptosis of tumor cells, but it could enhance the chemotherapeutic effect of other agents; the reason is that ABT-737 could increase expression of death signal factors. Thus, it displays synergis- tic effect with chemotherapeutics and radiation [25]. However, the clinical prospects of ABT-737 were hindered by its poor aqueous solubility and physiochemical properties. Then, ABT-263, a new inhibitor of Bcl-2 family proteins, was synthesized. The compound exhibited selective inhibition to Bcl-2-related tumor cells and elicited tumor regressions in B cell lymphoma and multiple myeloma xenograft mice. ABT-263 is investigated in clinical trials. Nevertheless, as preclinical data showed, ABT-263 induced concentrationdependent thrombocytopenia [26-30]. Souers et al. reported a new derivative of ABT-263 named ABT-199. ABT-199 exhibited highly potent, oral bioavailability with high affinity for Bcl-2 protein. More importantly, the compound inhibited the progress of $\mathrm{Bcl}-2$-dependent tumors, without reducing human platelets [31]. Mechanistic research revealed that the inhibitors could enhance the effects of the death signal, while displaying synergistic cytotoxicity with chemotherapeutic agents. Thereinto, ABT-737 showed a certain synergistic effect in combinational chemotherapy of HCC treatment. In order to overcome limitation of ABT-737 in liver cancer, Ren and colleagues demonstrated the ABT-737 and norcantharidin have synergistic effect in hepatocellular carcinoma cells which accordingly induces apoptosis [32]. Zheng and coworkers found that curcumin significantly enhanced inhibited effect of ABT-737 on liver cancer cells. And it also depends on the induction of apoptosis. Moreover, they indicated that activation of the ROS-ASK1-c-Jun N-terminal kinase pathway should be an important factor of the synergy in the combination [33]. Thus, ABT-199, ABT-263, and ABT-737 have potential to be used for screening of more effective drug combinations in treatment of HCC. Except for the effect of the drug by itself, another main barrier of combination chemotherapy is unifying the pharmacokinetics and cellular internalization of multiple drugs with a precise optimal ratio. The nanocarrier can resolve the issue of drug delivery [34].

Encapsulation and portioning of multiple therapeutic agents into a target nanocarrier system will provide a satisfactory strategy for synchronous delivery to the targeted tissues and cells $[35,36]$. Various studies focused on combinational agent nanocarriers for HCC delivery and treatment. Zhang and coworkers prepared a DOX and sorafenib-targeted codelivery NPs for treatment of HCC and showed efficient results in the antitumor experiment [37]. Another codelivery nanocarrier of DOX and curcumin was reported [38]. Besides, optimal treatment also depends on a reasonable ratio between drugs. However, the ratio of drugs in vehicles is too difficult to adjust. In our previous study, a multifunctional nanoparticle with an adjustable drug ratio and excellent delivery was developed. In the case, the loading of the two drugs in the nanoparticle, which are DOX and apogossypolone, met an optimal ratio by adjusting the amount of subsystems [39]. Based on the study, an excellent antiHCC nanocarrier was designed. Initially, we investigated the synergistic anti-HCC of DOX combined with ABT-199, ABT-263, and ABT-737. Then, coencapsulated optimal combination by CSaSt and HA via a self-assembly process formed DDS NPs. The NPs could effectively deliver the drugs into HCC cells in vitro and in vivo and implemented 


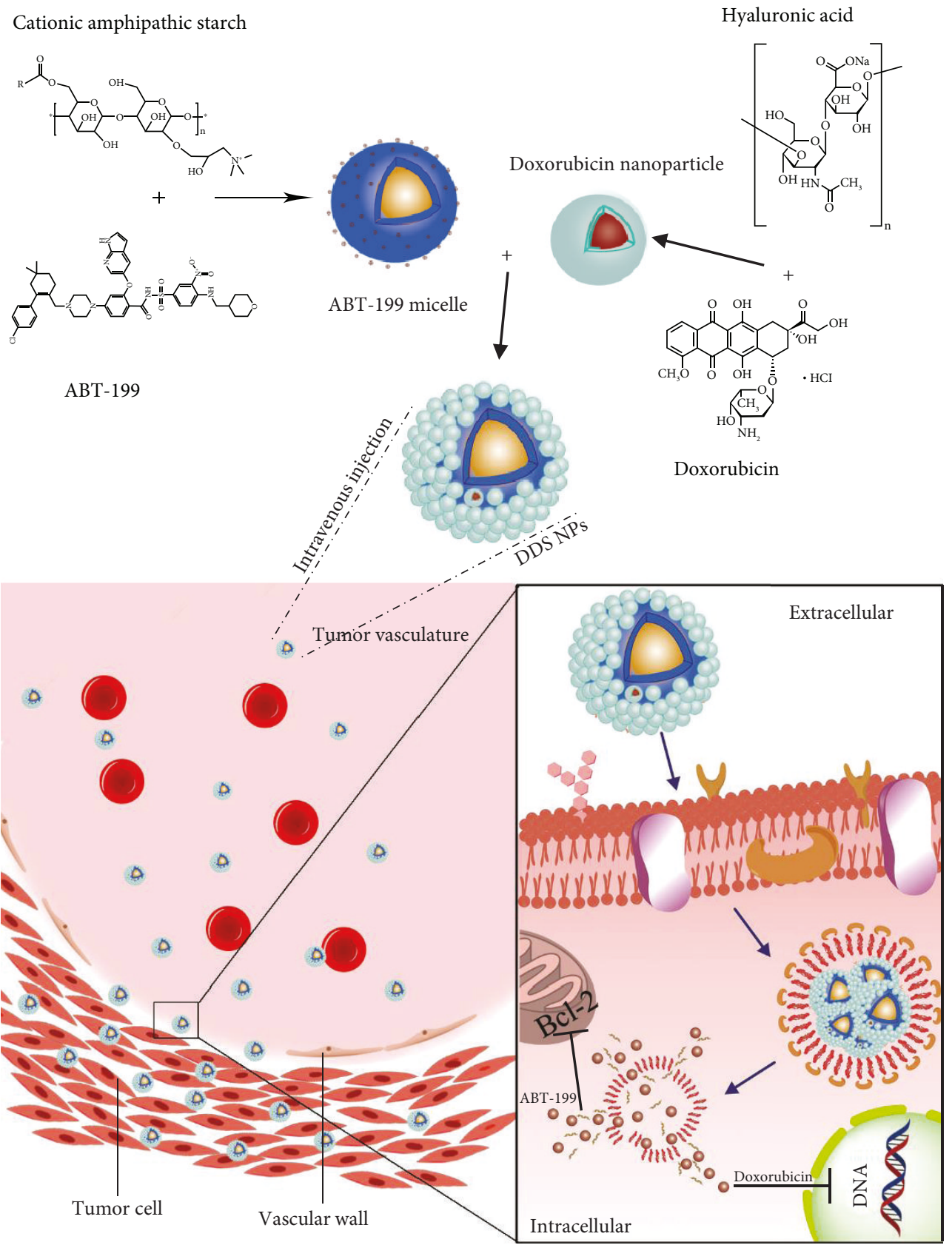

Scheme 1: Preparation, targeting, and internalization process of DDS NPs.

superior synergistic therapy against HCC cell lines and the HCC xenograft mouse model (Scheme 1). Therefore, the characteristics indicated that DDS NPs have great potential for the treatment of HCC.

\section{Materials and Methods}

2.1. Materials. CSaSt has been synthesized in our lab. HA (6.2 kDa), IR-780, DOX, ABT-199, ABT-263, ABT-737, and a WST-8 kit were supplied by Aladdin Inc. (Shanghai, China). Ampicillin, streptomycin, and coumarin-6 were purchased from Sigma-Aldrich (MO, USA). Fetal bovine serum (FBS), DMEM high glucose medium, and trypsin were bought from Thermo Fisher Scientific Corp. (Shanghai,
China). The cell culture plates, dishes, and other consumables were supplied by Corning Corp. (NY, USA). Other reagents were purchased from Haimeng Corp. (Xi'an, China). The Huh-7, BEL-7402, and SMMC-7721 cell lines were provided by Procell Life Science \& Technology Co., Ltd. (Wuhan, China). BALB/c mice and nude mice were purchased from the Department of Experimental Animals, Xi'an Jiaotong University.

2.2. Screening of Optimal Synergistic Drug Combination. Three HCC cell lines (BEL-7402, Huh-7, and SMMC-7721) were cultured in medium with $10 \%(v / v)$ of FBS, $50 \mu \mathrm{g} / \mathrm{L}$ of ampicillin, and $36 \mu \mathrm{g} / \mathrm{L}$ of streptomycin and incubated under $5 \% \mathrm{CO}_{2}$ at $37^{\circ} \mathrm{C}$ in an incubator (3111, Thermo Fisher, 
Shanghai, China). The WST assay was used for investigation of cell growth inhibition. The cells were treated with different concentrations of DOX, ABT-199, ABT-263, and ABT-737 for $72 \mathrm{~h}$. The concentrations of agents included DOX at $0.12-0.72 \mu \mathrm{mol} / \mathrm{L}, \mathrm{ABT}-199$ at $2.13-13.44 \mu \mathrm{mol} / \mathrm{L}, \mathrm{ABT}-263$ at $2.73-15.21 \mu \mathrm{mol} / \mathrm{L}$, and ABT-737 at $1.98-11.37 \mu \mathrm{mol} / \mathrm{L}$. The synergistic effects of DOX and ABT-199, ABT-263, and ABT-737 were assayed by two agents with different dilution ratios. The concentrations of drugs were determined by $\mathrm{IC}_{50}$, respectively. All of the samples had 3 duplicated wells during treatment. After treatment, $100 \mu \mathrm{L}$ of colorless medium, which contained $10 \%(v / v)$ of WST-8, was added to each well. After $2 \mathrm{~h}$ of incubation, the absorbances of wells were measured by the plate reader (Infinite ${ }^{\circledR} 200$ $\mathrm{PRO}$, Tecan, Switzerland) at $450 \mathrm{~nm}$. The $\mathrm{IC}_{50}$ values were calculated with GraphPad Prism 5 software. Drug combination effect was studied by CompuSyn software [40]. CI (combination index) values reflected combinable effect of drugs, where CI values less than 1.00 were considered as a synergistic effect.

2.3. Preparation and Characterizations of DDS NPs. The process of preparation included two steps. In the first step, the core ABT-199 (optimum drug combined with DOX)-loaded micelles were constructed. $10 \mathrm{mg}$ of ABT-199 and $20 \mathrm{mg}$ of CSaSt were dissolved into $1 \mathrm{~mL}$ of DMSO. Then, the mix was dropwise added into $5 \mathrm{~mL}$ distilled water under stirring and kept stirring for $15 \mathrm{~min}$. The micelle solution was dialyzed (7000 Da MWCO) with water for $24 \mathrm{~h}$, while replacing the water twice during dialysis. On the other side, $1 \mathrm{~mL}$ of DOX solution $(10 \mathrm{mg} / \mathrm{mL})$ was added into $5 \mathrm{~mL}$ of HA solution $(4 \mathrm{mg} / \mathrm{mL})$ under stirring. After $20 \mathrm{~min}$ of stirring, DOX NPs were obtained. Then, the ABT-199 micelle solution was slowly dropped to the DOX NPs solution. The proportion of the two kinds of solutions was based on the solvent mass ratio. After preexperiments, the realizable interval of proportions about ABT-199 MCs and DOX NPs was 1:8 to 15. For $30 \mathrm{~min}$ of electrostatic self-assembly, the DDS NPs were prepared successfully. The soluble impurities and solvent were removed by dialysis.

The Malvern instrument (Nano-ZS90, Malvern, UK) and transmission electron microscope (TEM) (JEM-2100F, JEOL, Japan) were used to measure size and morphology of DDS NPs. The drug loading (DL) and encapsulation efficiency (EE) of ABT-199 and DOX in DDS NPs were measured by HPLC and calculated with formulas

$$
\begin{gathered}
\text { Encapsulation efficiency }(\%)=\frac{C_{\text {drug remain }}}{C_{\text {drug input }}} \times 100 \%, \\
\text { Drug loading }(\%)=\frac{C_{\text {drug remain }}}{C_{\text {DDS NP input }}} \times 100 \% .
\end{gathered}
$$

2.4. In Vitro Release and Stability of DDS NPs. The drug releasing of DDS NPs was investigated by dialysis. DOX is primarily used in this test, because of having fluorescence and solubility. In comparison, ABT-199 is a hydrophobic molecule. It is too difficult to use in aqueous dialysis. As for the control groups, the same concentration of free DOX and DDS NPs with $10 \mathrm{U} / \mathrm{mL}$ of HAase was dialyzed under the same condition. The stability of DDS NPs at different times and temperatures was measured by changing the size.

2.5. In Vitro Cytotoxicity and Internalization of DDS NPs. The cytotoxicity of DDS NPs was investigated by the WST8 method as described previously. The medium with the drug or NPs was added into 96-well plates. There were 5 concentration gradients in each sample. The cellular internalization of the DDS NPs was observed by fluorescence NPs, in which coumarin- 6 was used to label DDS NPs. HA was utilized in the blocking test. The cells were cultured for $48 \mathrm{~h}$ and then added the samples at different times. The cells were fixed with paraformaldehyde (4\%) for $10 \mathrm{~min}$, and DAPI was added for labeling the nucleus. Observation was executed under a confocal microscope (TCS SP5 II, Leica, Germany).

2.6. In Vivo Toxicity of DDS NPs. In vivo toxicity was investigated by the acute toxicity method and the hemolysis assay. In the acute toxicity method, ten male and ten female mice were divided randomly into two groups to compare the in vivo toxicity of DDS NPs and free drug combination. The mice were fed in a SPF condition. After 3 days, the mice were intravenously injected drugs and the NPs with same dosages. The death number of mice were recorded, and mortality rates were counted within $14 \mathrm{~d}$. The liver and heart tissues were taken for histopathology analysis. For the hemolysis assay, the whole blood of mice was drawn and then treated with an anticoagulant. The blood was divided into 6 groups which are empty NPs, Triton X-100 (1\% v/v), DDS NPs (DOX: $0.01 \mathrm{mg} / \mathrm{mL}$, ABT-199: $0.1 \mathrm{mg} / \mathrm{mL}$ ), dual drugs (concentrations were the same with DDS NPs), DMSO $(0.5 \% v / v)$, and untreated group. The blood samples were incubated at $37^{\circ} \mathrm{C}$ for $2 \mathrm{~h}$. After incubation, the samples were centrifuged at $13000 \mathrm{rpm}$ for $15 \mathrm{~min}$; then, the supernatants were collected for investigation of lysis. The supernatants were measured by an ultraviolet-visible spectrophotometer (UV2900, SOPTOP, Shanghai, China) at $394 \mathrm{~nm}$ absorbance [41]. All of the animal experiments were approved by the Laboratory Animal Administration Committee of Xi'an Medical University.

2.7. HCC Animal Model. Male nude mice (4 w, approximately $16 \mathrm{~g}$ ) were fed in a SPF condition. Adaptation was for $1 \mathrm{w}$. About $100 \mu \mathrm{L}$ of DMEM medium with $5 \times 10^{5}$ of BEL-7402 cells were subcutaneously inoculated into the flank of the mouse. When tumors grew to the appropriate volume, the tumor-bearing mouse models could be used for further experiments.

2.8. In Vivo Distribution of DDS NPs. Four tumor-bearing mouse models were used for investigation of in vivo distribution of DDS NPs. $200 \mu \mathrm{L}$ of IR-780 loading DDS NPs was intravenously injected in an animal model; another one was treated with the same amount of free IR-780 as the control. At different time points, the fluorescent signals of IR-780 loading DDS NPs and free IR-780 in the mice were observed by a Carestream molecular imaging system (Carestream Health, NY, US). All mice were euthanized by $\mathrm{CO}_{2}$ overdose when imaging was finished, and the heart, liver, spleen, lung, 
TABLE 1: CI and dose reduction values for inhibition on BEL-7402, SMMC-7721, and Huh-7 by combining DOX with ABT-199.

\begin{tabular}{|c|c|c|c|c|c|c|c|}
\hline \multirow{3}{*}{$\%$ inhibition } & \multirow{3}{*}{$\mathrm{CI}$} & \multicolumn{3}{|c|}{ DOX } & \multicolumn{3}{|c|}{ ABT-199 } \\
\hline & & \multicolumn{2}{|c|}{ Conc. $(\mu \mathrm{mol} / \mathrm{L})$} & \multirow{2}{*}{ Dose reduction } & \multicolumn{2}{|c|}{ Conc. $(\mu \mathrm{mol} / \mathrm{L})$} & \multirow{2}{*}{ Dose reduction } \\
\hline & & Alone & Mix & & Alone & Mix & \\
\hline \multicolumn{8}{|l|}{ BEL-7402 } \\
\hline 50 & 0.6218 & 0.237 & 0.098 & 2.418 & 12.434 & 5.888 & 2.112 \\
\hline 75 & 0.8341 & 0.532 & 0.132 & 4.030 & 21.875 & 10.962 & 1.996 \\
\hline 95 & 1.0126 & 0.997 & 0.369 & 2.701 & 34.511 & 27.435 & 1.258 \\
\hline \multicolumn{8}{|l|}{ SMMC-7721 } \\
\hline 50 & 0.5832 & 0.384 & 0.127 & 3.024 & 13.822 & 5.917 & 2.336 \\
\hline 75 & 0.7421 & 0.711 & 0.218 & 3.261 & 24.329 & 9.998 & 2.433 \\
\hline 95 & 0.9735 & 1.203 & 0.435 & 2.766 & 37.096 & 23.842 & 1.558 \\
\hline \multicolumn{8}{|l|}{ Huh-7 } \\
\hline 50 & 0.7125 & 0.256 & 0.067 & 3.821 & 13.911 & 7.896 & 1.762 \\
\hline 75 & 0.8641 & 0.497 & 0.158 & 3.146 & 28.502 & 13.866 & 2.056 \\
\hline 95 & 0.9658 & 0.899 & 0.309 & 2.909 & 39.710 & 30.117 & 1.319 \\
\hline
\end{tabular}

kidney, and tumors were taken. The fluorescent signals in tissues were measured in the same conditions.

2.9. In Vivo Tumor Inhibition Effect of DDS NPs. Thirty of one tumor-bearing model were used for the investigation of in vivo tumor suppression. The models were distributed into five groups randomly; each group had six mice. The treatments included (1) saline, (2) ABT-199 separately, (3) DOX separately, (4) ABT-199 with DOX, and (5) DDS NPs. When the tumor grew to a sufficient volume, treatment began. Each group was intravenously injected twice per week for three weeks, and volume of injection was $200 \mu \mathrm{L}$ with the same dosages. The sizes of tumors and weights of the bodies were recorded continuously.

2.10. Statistical Analysis. The GraphPad Prism 5.0 software was used for calculating the data. The data were presented as the mean \pm SD of independent experiments. A $P$ value $<0.05$ was considered to have a statistical difference between the compared data.

\section{Results and Discussion}

3.1. Synergistic Effect of DOX with ABT-199, ABT-263, or $A B T-737$. In previous reports, ABT-737 and chemotherapeutic agents had the synergistic effect during HCC treatment $[32,33]$. However, there was not a report of ABT-263 and ABT-199 in the combinational chemotherapy of HCC. In order to obtain the drug combination with better synergistic effect in HCC treatment, DOX was executed combined with ABT-199, ABT-263, and ABT-737 via the WST-8 method in three HCC cell lines (BEL-7402, SMMC-7721, and Huh-7), which had high expression levels of the Bcl-2 family protein. They remained sensitive to DOX, where the $\mathrm{IC}_{50}$ values of DOX were less than $0.5 \mu \mathrm{mol} / \mathrm{L}$. However, there were different sensitivities of ABT-199, ABT-263, and ABT-737. The cytotoxicity of ABT-263 was the lowest in the three inhibitors, and $\mathrm{IC}_{50}$ values in BEL-7402, SMMC7721, and Huh-7 were 20.856, 24.986, and 23.454 $\mu \mathrm{mol} / \mathrm{L}$, respectively. ABT-737 showed the most sensitivity in the three cell lines, and the $\mathrm{IC}_{50}$ values were generally only half of ABT-263. The cytotoxicity of ABT-199 was very close to ABT-737 (Table 1 and Tables S1 and S2). Then, DOX combined with ABT-199, ABT-263, and ABT-737 was investigated. Increased cytotoxicity was observed in all of the three cell lines when the ratio of DOX and the Bcl-2 inhibitor was $1: 10$. The CI was calculated by the CompuSyn software. The CI values demonstrated that the interaction between DOX and ABT-199 in the three cell lines after treatment was under 1.0 (Table 1), indicating that these two drugs have superior synergistic effect. Although, ABT-737 had the most cytotoxicity in the three cell lines, the synergistic effect and dose reduction of the combination with DOX were less than DOX with ABT-199. Instead, there was an antagonistic effect between DOX and ABT-263. Further detailed studies were carried out with the combination of ABT-199 and DOX.

3.2. Preparation and Characterization of DDS NPs. There are two obstacles that impede combinational chemotherapy in clinical development. Except for the effect of the drug by itself, another main problem is unifying the pharmacokinetics and cellular internalization of multiple drugs with a precise optimal ratio [34]. Advances in nanocarriers have supported remarkable opportunities in combination chemotherapy. Many nanoplatforms have been used for drug delivery [42]. In our previous study, a kind of CSaSt was prepared. The material could encapsulate a hydrophobic compound in the core to form a nanomicelle with an electropositive surface. The micelle could absorb the electronegative substance or particle to achieve multiple assembly. Furthermore, another advantage of this method was that the two drugloading NPs were assembled with an adjustable ratio of synergy [39]. Based on this, the DOX and ABT-199 coencapsulation nanocarrier with an optimal drug ratio was designed and prepared. The process included two steps. First, the ABT-199-loaded micelles and DOX NPs were constructed, respectively. Then, we assembled DDS NPs from two 

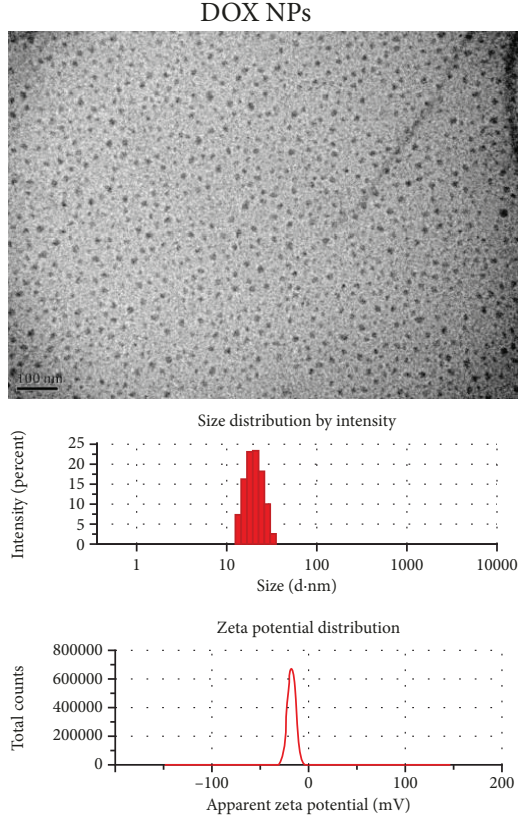

(a)

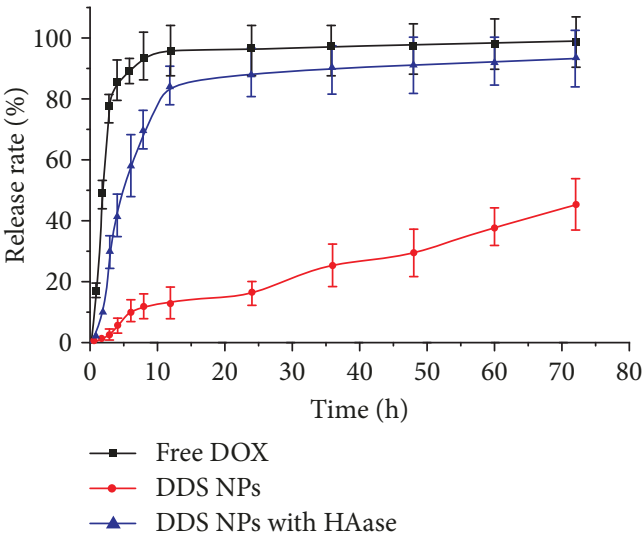

(d)
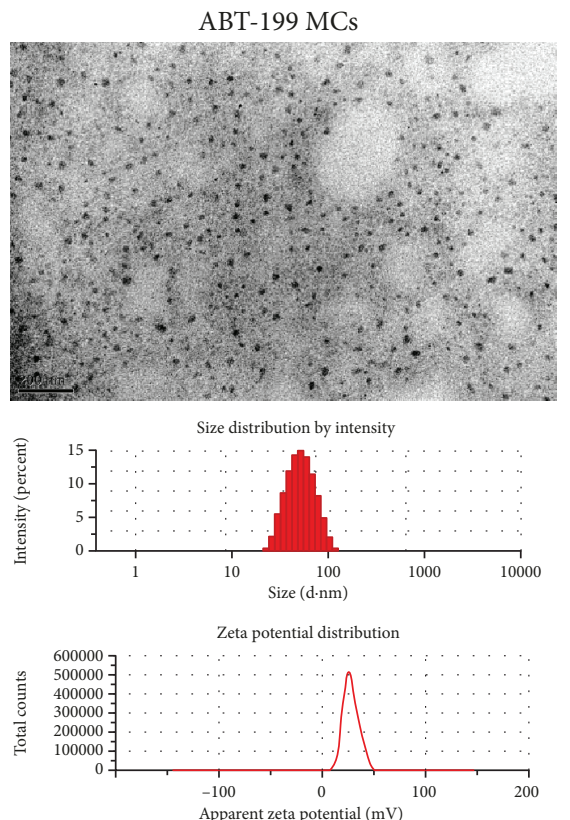

(b)

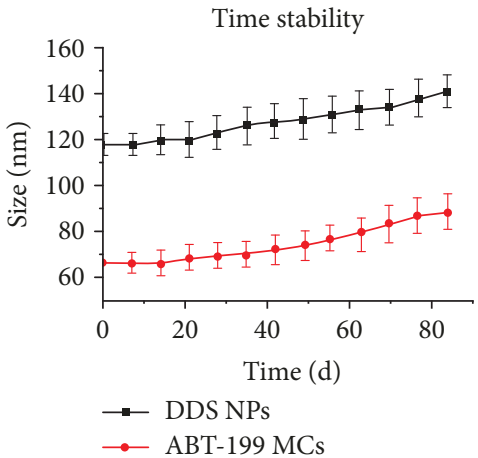

(e)
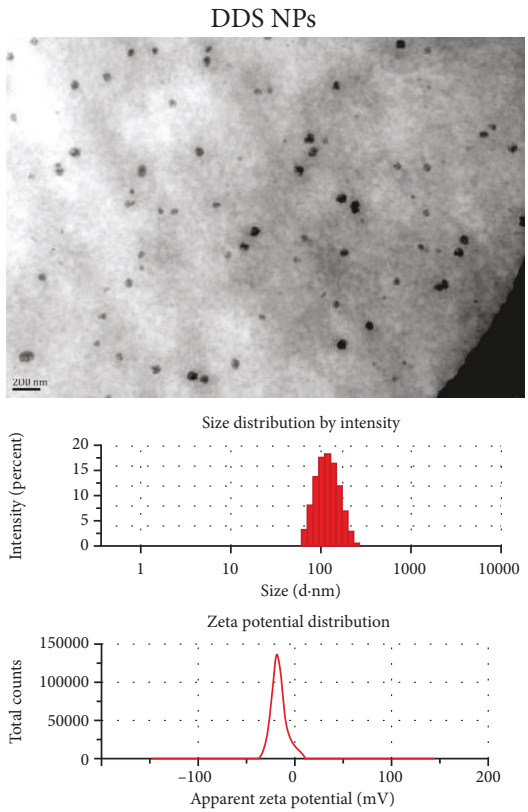

(c)

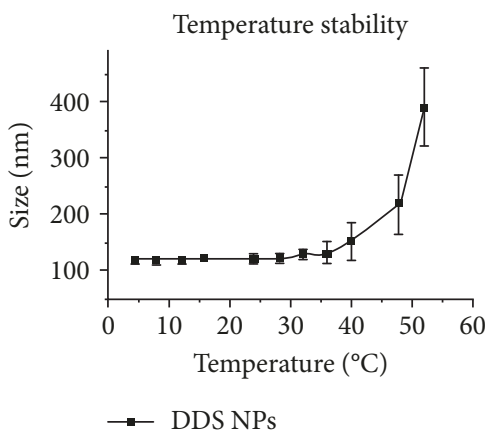

(f)

FIgURE 1: Characterization of DDS NPs: (a) morphology, size, and zeta potential of DOX NPs; (b) morphology, size, and zeta potential of ABT-199 micelles; (c) morphology, size, and zeta potential of DDS NPs; (d) in vitro DOX releasing of free DOX, DDS NPs, and DDS NPs with HAase; (e) in vitro long-term stability of ABT-199 micelles and DDS NPs; (f) in vitro temperature stability of ABT-199 micelles and DDS NPs. The data reported are the mean \pm SD for triplicate samples.

counterparts via electrostatic adsorption, where ABT-199loaded micelles served as the core and DOX NPs were absorbed around them. Figures 1(a)-1(c) show the TEM images of ABT-199-loaded micelles, DOX NPs, and DDS NPs. As shown in the photos, three kinds of NPs were monodispersed in aqueous solutions. The shapes of ABT-199loaded micelles and DOX NPs were spherical, and the average sizes of them were $82.3 \pm 6.9 \mathrm{~nm}$ and $21.4 \pm 4.1 \mathrm{~nm}$, respectively. Moreover, DDS NPs showed relative surface roughness, and the size was $112.6 \pm 13.4 \mathrm{~nm}$. All of the nanocarriers showed homogeneous size distribution, where PDI of DOX NPs, ABT-199 MCs, and DDS NPs were 0.342, 0.211 , and 0.237 , respectively. These results accorded with the expected outcome where DDS NPs were assembled by DOX NPs around ABT-199 micelles, and the shape was similar to a raspberry. The size of DDS NPs was below the lim- ited size for leaky pathological capillaries, which give NPs a potential feature for use in negatively targeted cancer chemotherapy via the enhance permeability and retention (EPR) effect [43]. The sizes of NPs were also consistent with TEM images. However, the NPs in measurements of dynamic light scattering were bigger than the TEM observation. The main reason was that the aqueous solvent was eliminated during the process of TEM observation. The zeta potentials proved the encapsulation process. The zeta potentials of ABT-199loaded micelles, DOX NPs, and DDS NPs were $+34.8 \pm$ $5.1,-24.1 \pm 3.5$, and $-26.1 \pm 6.7 \mathrm{mV}$, respectively. It further proved that DDS NPs could be attributed to the electrostatic absorption between positive ABT-199-loaded micelles and negative DOX NPs, and the latter covered the surface of the former. The negative zeta potentials of DDS NPs supported good dispersion stability in the circulatory system [44]. 
The capacities of DDS NPs showed a direct influence on drug delivery and synergistic effect. Thus, the EE and DL of DDS NPs were measured by the HPLC method and calculated. The maximal EE and DL of ABT-199 were $94.7 \pm 2.8 \%$ and $14.1 \pm 1.1 \%$, respectively; meanwhile, the EE and DL of DOX were $90.2 \pm 4.3 \%$ and $1.5 \pm 0.4 \%$, respectively, which meant that DDS NPs have remarkable encapsulation. Ulbrich et al. codelivered DOX and docetaxel into a core-shell polymer through self-assembly of the copolyester PBS/PBDL and HPMA-based copolymers. In this study, DL of DOX was similar with DDS NPs, but docetaxel loading is only $5.5 \%$ [45]. Lv et al. reported a PEG-polypeptide nanovehicle, where drug loading was about $8 \%$ in total [46]. Furthermore, the data indicated that the ratio of $\mathrm{ABT}-199$ and $\mathrm{DOX}$ was about 1:10, which was matched with the optimal rate of combination. As shown in Figure 1(d), the release profiles of DOX in DDS NPs were investigated in aqueous condition, free DOX, and DDS NPs with HAase as the control. Free DOX showed obvious burst releasing in the dialysis; it was rapidly released from the dialysis membrane into the outer phase, with more than $95 \%$ of the solvent burst released within $12 \mathrm{~h}$. In contrast, only less than $50 \%$ of DOX in DDS NPs was released out after $72 \mathrm{~h}$. It is noteworthy that HAase could increase releasing of DOX from DDS NPs. When the HAase was added, more than $80 \%$ of DOX was released rapidly. It is because the HA layer degraded progressively. The result shows that DDS NPs could encapsulate hydrosoluble DOX via electrostatic interaction with $\mathrm{HA}$, and the drug was released effectively in tumor tissues, where there was a higher concentration of HAase in many solid tumor tissues than normal tissues. ABT-199 is water-insoluble. The amount in DDS NPs was determined in residues of lyophilization after dialysis. More than $95 \%$ of ABT-199 remained in DDS NPs, which means that encapsulation of ABT-199 was very stable. The changes of hydrodynamic diameter measurements were investigated for stabilities. The sizes of ABT-199 micelles and DDS NPs were stable at $90 \mathrm{~d}$, which were only less than $10 \%$ of increase (Figure 1(e)). Therefore, DDS NPs could remain very stable in neutral $\mathrm{pH}$ and $4^{\circ} \mathrm{C}$. Figure $1(\mathrm{f})$ shows the temperature stability of DDS NPs. When the temperature was below $40^{\circ} \mathrm{C}$, both of ABT-199 MCs and DDS NPs were very stable. However, if the temperature exceeded $40^{\circ} \mathrm{C}$, the size and dispersity decreased rapidly. It was attributed to the electrostatic interaction significantly affected by thermal force. Moreover, the thermodynamic factor is the key in the formation of micelles. The process is associated with compensation of entropy and enthalpy and related to the temperature. Temperature influences movement of the hydrophobic chain at temperatures above their glass transition temperature [47].

3.3. In Vitro Cytotoxicity and Cellular Internalization Behavior of DDS NPs. Even though the synergistic effect of DOX and ABT-199 was demonstrated, DDS NPs retaining the optimal effect of inhibition remains unknown. Therefore, in vitro cytotoxicity of DDS NPs in the three HCC cell lines was investigated firstly. As Figure S1 shows, empty NPs exhibited little toxicity indicating safety of the materials in

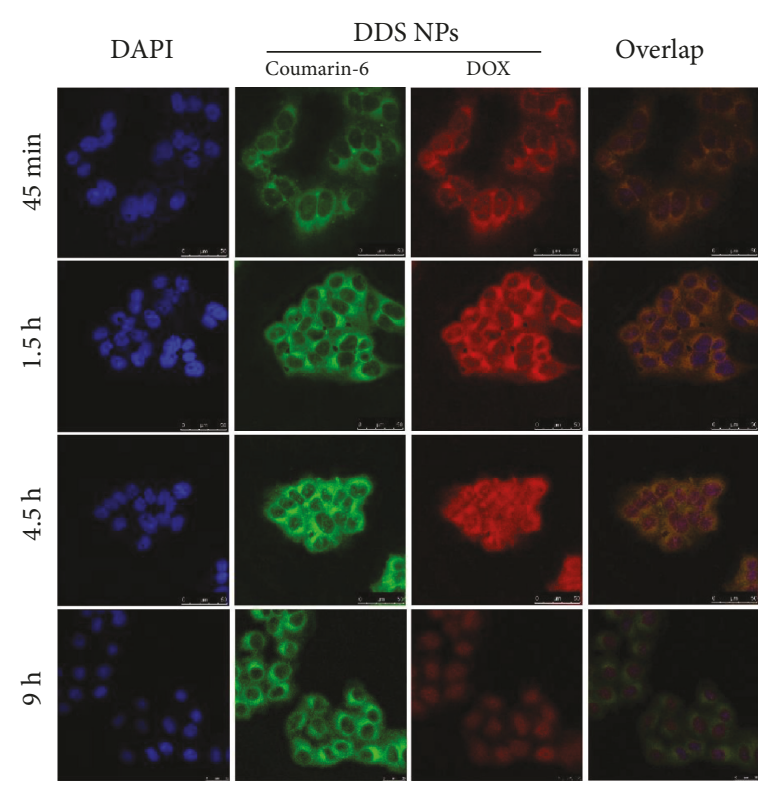

FIgURE 2: In vitro internalization of DDS NPs. The BEL-7402 cells were incubated with dual-fluorescence DDS NPs for $45 \mathrm{~min}, 1.5 \mathrm{~h}$, $4.5 \mathrm{~h}$, and $9 \mathrm{~h}$. The laser excitations were 358,458 , and $514 \mathrm{~nm}$, respectively.
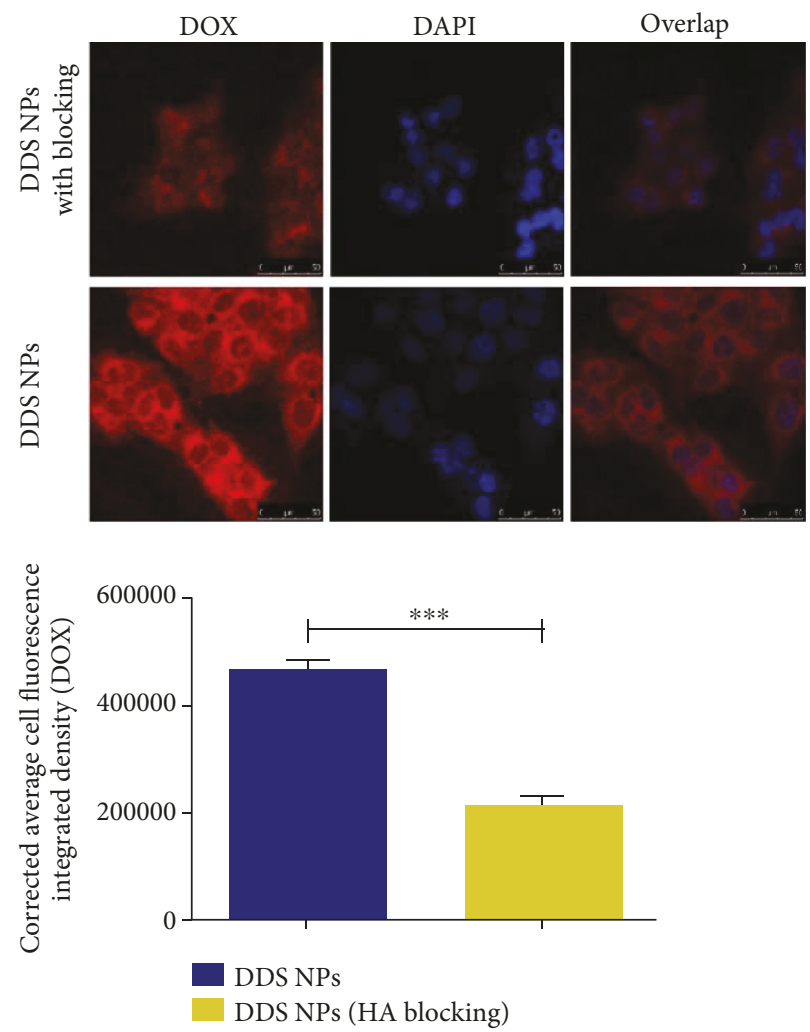

FIgURE 3: In vitro cell uptake behavior of DDS NPs. The fluorescent images of DDS NPs showing their amount in the presence of HA as a blocking agent in BEL-7402 cells. Cell nucleus stained with DAPI. Cells containing DDS NPs were colored red (DOX). Histogram showed cell fluorescence integrated density of DDS NPs and DDS NPs with hyaluronic acid blocking. The data reported are the mean \pm SD for triplicate samples; ${ }^{* * *} P<0.001$. 


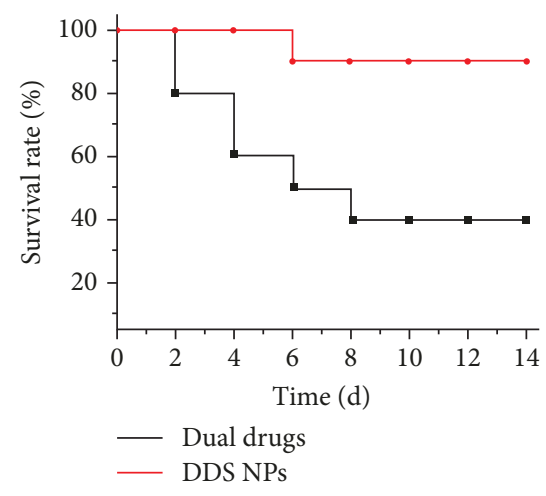

(a)

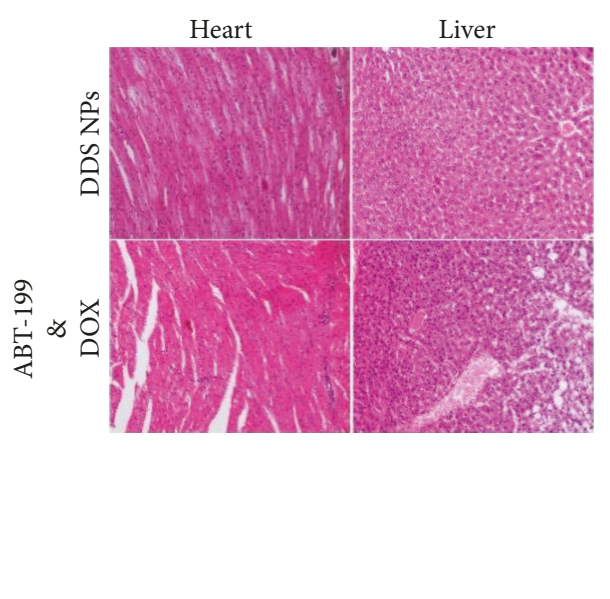

(b)

FIGURE 4: In vivo toxicity results of DDS NPs: (a) survival rate of free dual drugs and DDS NPs, there were 10 mice in each group; (b) the results of pathological examination of the heart and liver treated by dual drugs and DDS NPs. HE stain was used. Magnification was 200x.

subsequent experiments. The cytotoxicity of DDS NPs in cell lines was similar with the free drug combination. Thus, the in vitro cytotoxicity of DDS NPs was all due to the drugs which were coencapsulated in the NPs. The results were matched with other combined nanocarriers reported [48-50].

In order to observe the process of internalization and intracellular release of DDS NPs. The fluorescent dye was utilized in the experiments. Coumarin-6 is a hydrophobic green fluorescent dye which has a high quantum yield, stable fluorescence, and strong affinity to the membrane. It is widely used as a model compound loaded in solid lipid NPs [51, 52]. The dye was used to encapsulate in the core of DDS NPs; in addition, DOX has red fluorescence; therefore, dual-fluorescence DDS NPs were obtained. Figure 2 shows the process of internalization of DDS NPs in the BEL-7402 cell line. The concentrations of dyes increased with extended treatment time and reached a peak in $4.5 \mathrm{~h}$ after adding NPs. However, dynamic distributions of DOX and coumarin-6 exhibited significant distinction during the process of internalization. In the initial $1.5 \mathrm{~h}$, both DOX and coumarin- 6 were mainly accumulated in the cytoplasm. The intensity of coumarin- 6 was primarily retained in the cytoplasm during the overall process. The fluorescent signal of DOX, in contrast, had an obvious shift later on. After $4.5 \mathrm{~h}$, the distribution of DOX diffused from the cytoplasm into the entire cell. $9 \mathrm{~h}$ later, majority of DOX gathered in the nucleus. This phenomenon indicated that intracellular release of loading occurs in multiple steps. Initially, DDS NPs entered the cytoplasm via endocytosis. At this stage, the NPs were contained within the endosomes. The endosomes were transformed to lysosomes via acidification over time. Then, DDS NPs began to break down, and HA was decomposed, followed by the release of DOX. DOX primarily binds to the nucleus; thus, the fluorescent signal was shifted from the cytoplasm into the nucleus. In the second step, the micelle core was exposed in the lysosomes, and the materials, which were based on starch, were decomposed causing coumarin- 6 to be released. Coumarin- 6 is a hydrophobic dye and has high affinity to the membrane. Thus, green fluorescence remained in the cytoplasm. The result suggested that DDS NPs could perform intracellular delivery of dual drugs and exhibit continuous release of the drugs into different targets. Thus, the synergy of DOX and ABT-199 could be guaranteed. Figure 3 shows the cellular uptake profiles with or without HA blocking. In this case, $1 \%$ of HA was added into the medium before the incubation with DDS NPs. An evident fluorescent signal could be observed in the cytoplasm after $1 \mathrm{~h}$ of incubation. In contrast, only slight fluorescence was observed during the blocking treatment at the same time, which means that HA molecules competed with DDS NPs in the process of internalization. The result indicated that the endocytosis of DDS NPs was mediated by HA receptor which is on the surface of the cell. HA is a receptor of the $\mathrm{CD} 44$ protein, which has a high expression on the surface of the hepatic cancer cell. It can target tumor tissues [53].

3.4. In Vivo Toxicity of DDS NPs. In vivo toxicity is one of the most important measurement indices in the study of antitumor drug delivery. One primary advantage of antitumor nanocarriers is a decrease in nonspecific toxicity in normal organs and tissues. Thus, in vivo acute toxicity in mice was used to investigate systemic toxicity. Figure 4 shows the results of acute toxicity of DDS NPs and free dual drugs. There was only 1 mouse dead in the DDS NPs treatment group. In the free drug group, only $40 \%$ of the mice survived. The result demonstrated that acute toxicity of DDS NPs was significantly lower than the free drugs. After $14 \mathrm{~d}$, the surviving mice were euthanized, and their heart and liver were pathologically sectioned and HE-stained. DOX proved to have cardiac toxicity. As a hydrophobic compound, ABT-199 is primarily metabolized in the liver. The pathological investigation of the heart and liver demonstrated a similar result. The tissues from the heart and liver in the dual-drug group showed obvious pathological changes. The tissue of the heart exhibited typical characteristics of myocarditis. As shown in the right edge of the heart image in dual- 

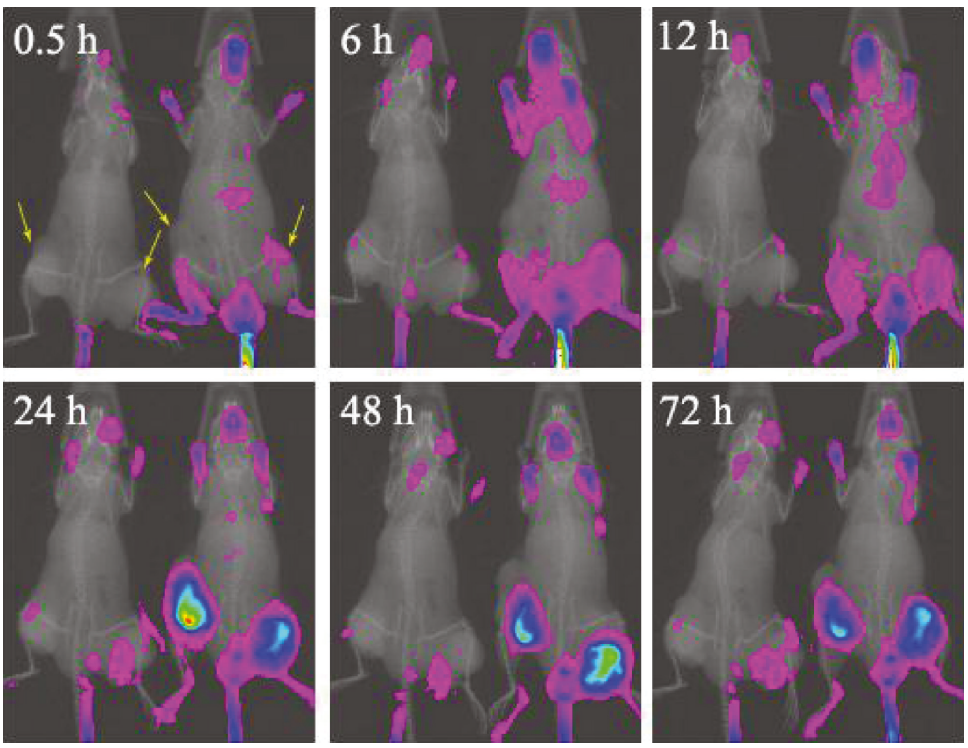

(a)

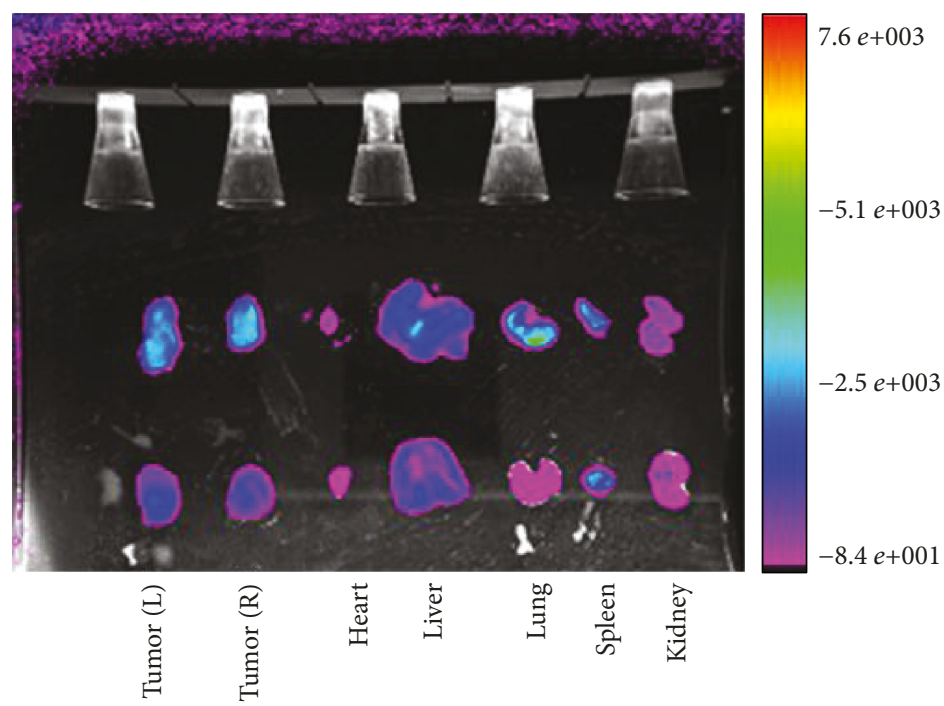

(b)

FIGURE 5: In vivo distribution of DDS NPs. In each picture, the right mouse was injected IR-780-loaded DDS NPs; meanwhile, the left mouse was injected free IR-780 dye. And then, in vivo distribution of NIR fluorescence was observed at different times. The arrows were pointed to tumors. In (b) were in vivo tissue distribution results of mice after injection for $72 \mathrm{~h}$.

drug treatment, in the part of the tissues, myocardial cells disappeared and were replaced by lymphocytes. Basophilic staining of the remaining myocardial cells in the inflammatory foci was enhanced. The pathological changes were also observed in hepatic tissues. Hepatocytes occurred swelling and irregular arrangement. Liver steatosis resulted in hepatic sinus stenosis and lipid droplet vacuoles in the cytoplasm of some hepatic cells. In contrast, the organs from the DDS NPs group were healthier than in the dual-drug group after treatment. The results of the hemolysis assay are shown in Figure S2. The lysis percentages in DDS NPs and empty NPs were $14 \%$ and $8 \%$, respectively. The data in dual drugs and DMSO groups showed higher lysis percentages, which were $38 \%$ and $11 \%$, respectively. The result indicated that DDS NPs markedly reduced the in vivo toxicity of the drugs.
3.5. In Vivo Distribution and Antitumor Effect of DDS NPs. An in vivo visual imaging technique uses fluorescently tagged nanocarriers injected into the animals that were diffused in the blood stream and channeled to the tissues. The nearinfrared fluorescent dyes have prominent superiority in animal imaging. IR-780 is a hydrophobic near-infrared fluorescent dye [54]. Thus, the dye was used to replace ABT-199 in the core of DDS NPs; therefore, near-infrared (NIF) fluorescent DDS NPs were obtained. The xenograft mouse models, which bore the BEL-7402 tumor, were used for investigating the in vivo distribution.

Figure 5 exhibits the in vivo NIF fluorescent images at different time points after injection. The arrow points to the tumors. In the free IR-780 group, the tumors only accumulated a small amount of fluorescent signals. At $0.5 \mathrm{~h}$, the 


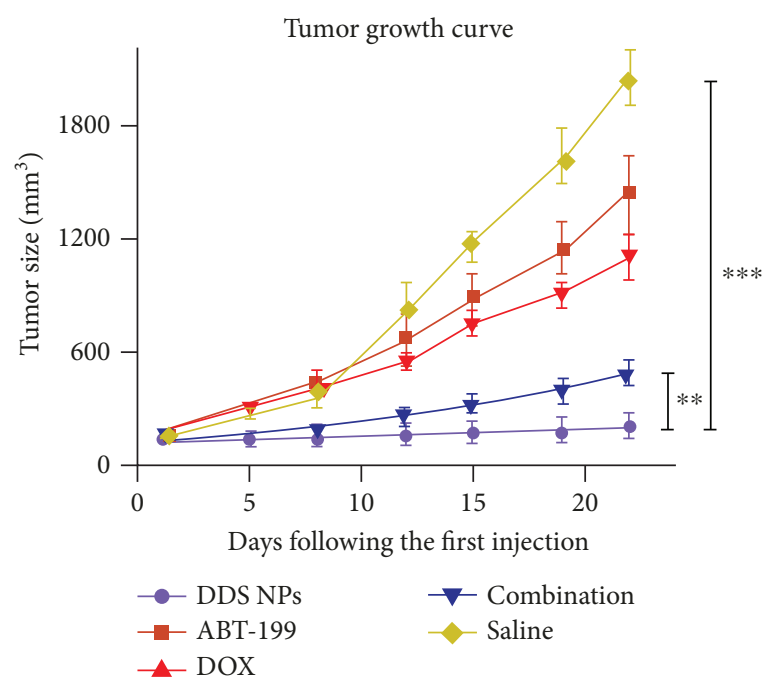

(a)

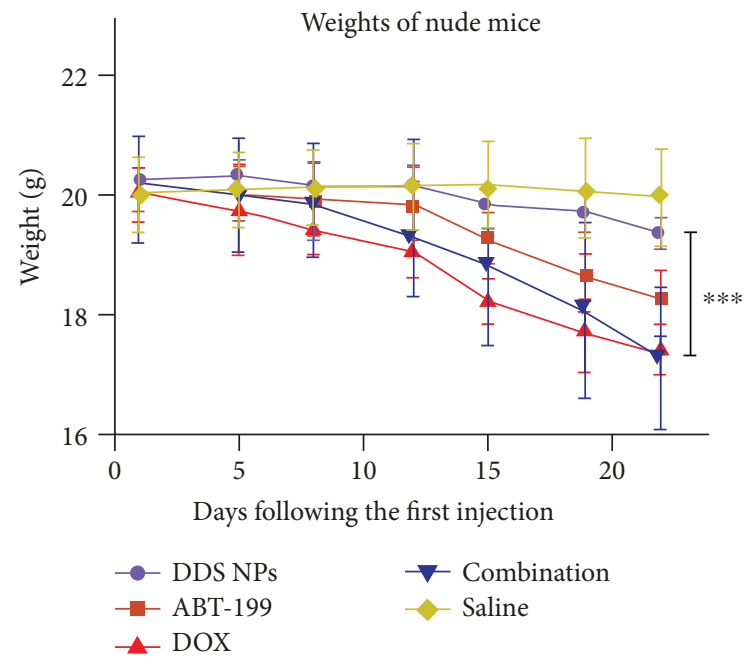

(b)

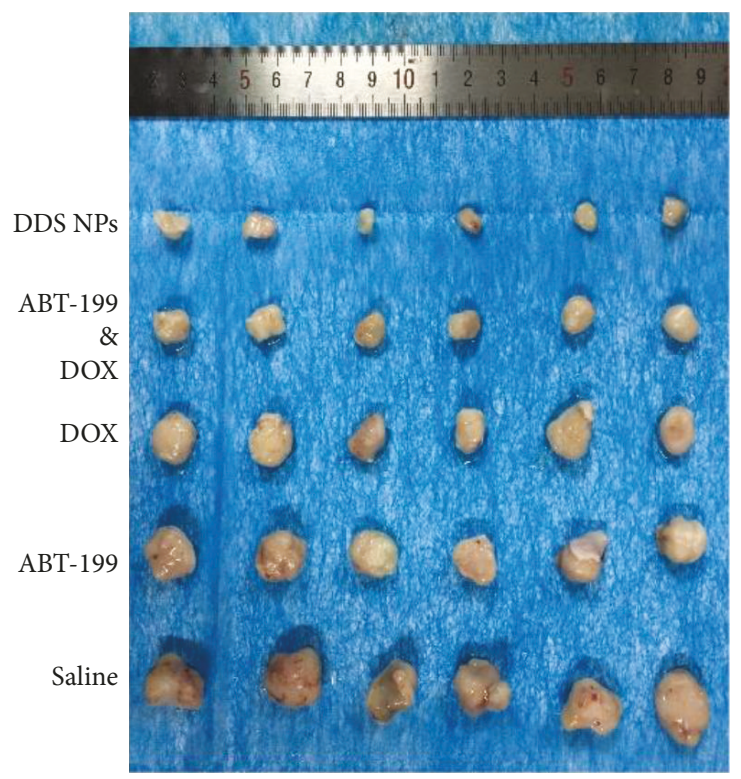

(c)

FIGURE 6: In vivo tumor inhibition of DDS NPs. (a) Volume curves of tumors in each treatment groups. (b) The body weight curves of mice in each group. (c) The tumors in different groups were taken out after euthanasia. Nude mice were transplanted tumors on the left flank. The treatment dosages of ABT-199 and DOX were $10 \mathrm{mg} / \mathrm{kg}$ and $1 \mathrm{mg} / \mathrm{kg}$, respectively. Each treatment was intravenously injected two times per week for three weeks. The tumorous volumes and body weights were recorded on the next day after injection. Each group has 6 mice, and error bars represent $\mathrm{SD} ;{ }^{* *} P<0.001$ and ${ }^{* *} P<0.01$.

fluorescent signal was accumulated in the liver of the mouse in the DDS NPs group. At $6 \mathrm{~h}$, the fluorescent signal started to accumulate in the tumor and tapered off from the liver. The signal peaked from 24 to $48 \mathrm{~h}$. Whereafter, the fluorescence remained $72 \mathrm{~h}$. The fluorescent signal in the mouth, urethral orifice, anus, and feet of the mice was due to the licking and touching of urine which contained the eliminated IR-780. The dye was hydrophobic, which allowed it to conveniently stick to the skin and mucous membranes [39]. The tumor and organs were collected after $72 \mathrm{~h}$. In the free IR-780 group (in bottom row), the fluorescent signal was mainly remained in the liver, spleen, and tumors. The signal in the DDS NPs group (in upper row) was much stronger than that in the free dye treatment. It was mainly accumulated in the tumors, lungs, spleen, and liver. The result indicated that DDS NPs significantly prolonged the in vivo circulation time of IR-780, and it was relatively strong in tumor tissues. It indicated that DDS NPs could improve the tissue distribution and support tumor targeting. The primary reason of DDS NPs accumulating in the liver, lung, and spleen was that these organs also have a $\mathrm{HA}$ receptor [53]. Thus, DDS NPs had a significant tumor targeting site and reduced the accumulation in normal tissues, which could improve the antitumor effect; meanwhile, it decreases the side effects of the drugs. 
Tumorous volume was intuitively responsive of in vivo antitumor effect. Thus, the tumor sizes and body weights of xenograft mice were measured after injection. The dosages of DOX and ABT-199 injection were 1 and $10 \mathrm{mg} / \mathrm{kg}$, respectively. The drug dose in the combination groups was the same as the single-drug treatment, which was matched with an optimal ratio during the preparation of the DDS NPs. The tumor growth curves are shown in Figure 6(a). The average volume of tumors in the saline group enlarged about 16 -fold. Moreover, the tumorous volumes in the free drug treatment groups showed different increasing curves over time. Among them, the tumor sizes in the group treated with a combination of drugs were less than the single-drug groups. The tumors in the DDS NPs group were much smaller than those of the other groups. After statistical calculation, (1) drugs and DDS NPs treatment groups had a significant effect compared to the saline group $(P<0.05)$, (2) the treatment with combination of drugs was more effective than both of the single-drug treatment groups $(P<0.001)$, and (3) the DDS NPs group showed a more notable tumorsuppressed effect than all of the free drug groups $(P<0.05)$. The in vivo tumor suppressive efficacy of DDS NPs was attributed to prolonged circulation, the effective tumor targeting, and synchronized delivery of the drugs with an optimal ratio. Compared with another study of anti-HCC, DDS NPs showed more effectiveness in a same BEL-7402 tumor-bearing mouse model [55]. The weights of mice between groups showed significant differences. All of the free drug treatment groups showed obvious descends when compared to the saline and DDS NPs groups. Among them, the weights in DOX and combination groups were the lightest. There were many reports suggesting that DOX have very high toxicity [56]. However, ABT-199 showed relatively low toxicity. The weights of mice in the DDS NPs group did not show significant difference in the saline group. The data indicated which DDS NPs could reduce drug toxicity, and the possible reason could be tumor targeting, enhanced circulation of the drugs, and sustained release in vivo.

\section{Conclusions}

In conclusion, we screened an excellent drug combination for the treatment of HCC, which included DOX and ABT199. We then prepared superior bioavailable, synergistic, targeted, and effective dual-drug nanocarriers, DDS NPs, which also have adjustable proportions of drugs, low acute toxicity, and sustained release performances. Compared with single DOX and ABT-199, DDS NPs effectively integrate the synergistic antitumor effect of dual drugs, and overcome in vivo toxicity of the drugs. The results indicated DDS NPs have great potential for clinical prospect of HCC chemotherapy.

\section{Abbreviations}

Bcl: B cell lymphoma

CI: Combination index

CSaSt: Cationic amphipathic starch

DDS: Dual-drug synergistic
DL: Drug loading

DOX: Doxorubicin

EE: Encapsulation efficiency

EPR: Enhanced permeability and retention

FBS: Fetal bovine serum

FDA: Food and Drug Administration

HA: Hyaluronic acid

HCC: Hepatocellular carcinoma

NIH: National Institutes of Health

NPs: Nanoparticles

PIAF: Doxorubicin versus cisplatin/interferon alpha2b/doxorubicin/fluorouracil

TEM: Transmission electron microscope.

\section{Data Availability}

The data used to support the findings of this study are available from the corresponding author upon request.

\section{Conflicts of Interest}

The authors declare no conflict of interest.

\section{Authors' Contributions}

Yun Zhou and Ke Li contributed equally to this work.

\section{Acknowledgments}

The authors would like to thank Yufeng Zhao and Yingguang Liu for cellular imaging of the study. This work was funded, in part, by the National Natural Science Foundation of China under Grant No. 81801863, the Research Project of Education Department of Shaanxi Provincial Government of Shaanxi Province of China under No. 18JK0679, the Research Foundation of Shaanxi Key Laboratory of Ischemic Cardiovascular Disease in Shaanxi Province of China under Grant No. 2017ZDKF05, the Natural Science Basic Research Plan in Shaanxi Province of China under Grant No. 2017JM8057, the Natural Science Basic Research Plan in Ningbo of China under Grant No. 2017A610144, and the Science and Technology Co-ordination and Innovation Project in Shaanxi Province of China under Grant No. 2016KTCQ03-07.

\section{Supplementary Materials}

Tables S1 and S2: CI and dose reduction values for inhibition of BEL-7402, SMMC-7721, and Huh-7 by combining DOX with ABT-263 or ABT-737. The synergistic effects of these two combinations were less than combined DOX with ABT-199. Especially in ABT-263 combined with DOX, it showed few synergy in three HCC cell lines. Figure S1: WST assay results of BEL-7402, SMMC-7721, and Huh-7 cells treated with dual drugs and DDS NPs. As the result show, the toxicity of empty NPs was almost none, and DDS NPs and free dual drugs have equal antiproliferating effect against three HCC cell lines in vitro. The result indicated that the DDS NPs could perform the synergistic anti-HCC effect of ABT-199 combined with DOX. (Supplementary Materials) 


\section{References}

[1] J. Ferlay, I. Soerjomataram, R. Dikshit et al., "Cancer incidence and mortality worldwide: sources, methods and major patterns in GLOBOCAN 2012," International Journal of Cancer, vol. 136, no. 5, pp. E359-E386, 2015.

[2] E. G. Giannini, F. Farinati, F. Ciccarese et al., "Prognosis of untreated hepatocellular carcinoma," Hepatology, vol. 61, no. 1, pp. 184-190, 2015.

[3] S. P. Choo, W. L. Tan, B. K. P. Goh, W. M. Tai, and A. X. Zhu, "Comparison of hepatocellular carcinoma in Eastern versus Western populations," Cancer, vol. 122, no. 22, pp. 34303446, 2016.

[4] P. Roxburgh and T. R. J. Evans, "Systemic therapy of hepatocellular carcinoma: are we making progress?," Advances in Therapy, vol. 25, no. 11, pp. 1089-1104, 2008.

[5] J. M. Llovet, S. Ricci, V. Mazzaferro et al., "Sorafenib in advanced hepatocellular carcinoma," New England Journal of Medicine, vol. 359, no. 4, pp. 378-390, 2008.

[6] A. L. Cheng, Y. K. Kang, Z. Chen et al., "Efficacy and safety of sorafenib in patients in the Asia-Pacific region with advanced hepatocellular carcinoma: a phase III randomised, doubleblind, placebo-controlled trial," The Lancet Oncology, vol. 10, no. 1, pp. 25-34, 2009.

[7] C. L. M. Olweny, T. Toya, E. Katongole-Mbidde, J. Mugerwa, S. K. Kyalwazi, and H. Cohen, "Treatment of hepatocellular carcinoma with adriamycin. Preliminary communication," Cancer, vol. 36, no. 4, pp. 1250-1257, 1975.

[8] J. Gehl, M. Boesgaard, T. Paaske, B. V. Jensen, and P. Dombernowsky, "Combined doxorubicin and paclitaxel in advanced breast cancer: effective and cardiotoxic," Annals of Oncology, vol. 7, no. 7, pp. 687-693, 1996.

[9] A. Kato, M. Miyazaki, S. Ambiru et al., "Multidrug resistance gene (MDR-1) expression as a useful prognostic factor in patients with human hepatocellular carcinoma after surgical resection," Journal of Surgical Oncology, vol. 78, no. 2, pp. 110-115, 2001.

[10] W. Jiang, Z. Lu, Y. He, and R. B. Diasio, "Dihydropyrimidine dehydrogenase activity in hepatocellular carcinoma: implication in 5-fluorouracil-based chemotherapy," Clinical Cancer Research, vol. 3, no. 3, pp. 395-399, 1997.

[11] Y. Soini, N. Virkajarvi, H. Raunio, and P. Paakko, "Expression of P-glycoprotein in hepatocellular carcinoma: a potential marker of prognosis," Journal of Clinical Pathology, vol. 49, no. 6, pp. 470-473, 1996.

[12] R. B. Weiss, "The anthracyclines: will we ever find a better doxorubicin?," Seminars in Oncology, vol. 19, no. 6, pp. 670686, 1992.

[13] H. BROXTERMAN and N. GEORGOPAPADAKOU, "Anticancer therapeutics: "Addictive" targets, multi-targeted drugs, new drug combinations,” Drug Resistance Updates, vol. 8, no. 4, pp. 183-197, 2005.

[14] F. Greco and M. J. Vicent, "Combination therapy: opportunities and challenges for polymer-drug conjugates as anticancer nanomedicines," Advanced Drug Delivery Reviews, vol. 61, no. 13, pp. 1203-1213, 2009.

[15] J. Lee, J. O. Park, W. S. Kim et al., "Phase II study of doxorubicin and cisplatin in patients with metastatic hepatocellular carcinoma," Cancer Chemotherapy and Pharmacology, vol. 54, no. 5, pp. 385-390, 2004.
[16] T. W. Leung, Y. Z. Patt, W. Y. Lau et al., "Complete pathological remission is possible with systemic combination chemotherapy for inoperable hepatocellular carcinoma," Clinical Cancer Research, vol. 5, no. 7, pp. 1676-1681, 1999.

[17] T. W. T. Leung, A. M. Y. Tang, B. Zee et al., "Factors predicting response and survival in 149 patients with unresectable hepatocellular carcinoma treated by combination cisplatin, interferon-alpha, doxorubicin and 5-fluorouracil chemotherapy," Cancer, vol. 94, no. 2, pp. 421-427, 2002.

[18] W. Yeo, T. S. Mok, B. Zee et al., "A randomized phase III study of doxorubicin versus cisplatin/interferon $\alpha$-2b/doxorubicin/fluorouracil (PIAF) combination chemotherapy for unresectable hepatocellular carcinoma," Journal of the National Cancer Institute, vol. 97, no. 20, pp. 1532-1538, 2005.

[19] D. W. Kim, C. Talati, and R. Kim, "Hepatocellular carcinoma (HCC): beyond sorafenib-chemotherapy," Journal of Gastrointestinal Oncology, vol. 8, no. 2, pp. 256-265, 2017.

[20] V. Lacronique, A. Mignon, M. Fabre et al., "Bcl-2 protects from lethal hepatic apoptosis induced by an anti-Fas antibody in mice," Nature Medicine, vol. 2, no. 1, pp. 80-86, 1996.

[21] E. Chun and K.-Y. Lee, "Bcl-2 and Bcl-xL are important for the induction of paclitaxel resistance in human hepatocellular carcinoma cells," Biochemical and Biophysical Research Communications, vol. 315, no. 3, pp. 771-779, 2004.

[22] M. Takahashi, H. Saito, K. Atsukawa, H. Ebinuma, T. Okuyama, and H. Ishii, "Bcl-2 prevents doxorubicininduced apoptosis of human liver cancer cells," Hepatology Research, vol. 25, no. 2, pp. 192-201, 2003.

[23] S. Wang, D. Yang, and M. E. Lippman, "Targeting Bcl-2 and Bcl- $\mathrm{X}_{\mathrm{L}}$ with nonpeptidic small-molecule antagonists," Seminars in Oncology, vol. 30, Supplement 16, pp. 133-142, 2003.

[24] J. X. Mi, G. F. Wang, H. B. Wang et al., "Synergistic antitumoral activity and induction of apoptosis by novel pan Bcl-2 proteins inhibitor apogossypolone with adriamycin in human hepatocellular carcinoma," Acta Pharmacologica Sinica, vol. 29, no. 12, pp. 1467-1477, 2008.

[25] T. Oltersdorf, S. W. Elmore, A. R. Shoemaker et al., "An inhibitor of Bcl-2 family proteins induces regression of solid tumours," Nature, vol. 435, no. 7042, pp. 677-681, 2005.

[26] C. Tse, A. R. Shoemaker, J. Adickes et al., “ABT-263: a potent and orally bioavailable Bcl-2 family inhibitor," Cancer Research, vol. 68, no. 9, pp. 3421-3428, 2008.

[27] C. M. Park, M. Bruncko, J. Adickes et al., "Discovery of an orally bioavailable small molecule inhibitor of prosurvival B-cell lymphoma 2 proteins," Journal of Medicinal Chemistry, vol. 51, no. 21, pp. 6902-6915, 2008.

[28] W. H. Wilson, O. A. O'Connor, M. S. Czuczman et al., "Navitoclax, a targeted high-affinity inhibitor of BCL-2, in lymphoid malignancies: a phase 1 dose-escalation study of safety, pharmacokinetics, pharmacodynamics, and antitumour activity," Lancet Oncology, vol. 11, no. 12, pp. 1149-1159, 2010.

[29] A. W. Roberts, J. F. Seymour, J. R. Brown et al., "Substantial susceptibility of chronic lymphocytic leukemia to BCL2 inhibition: results of a phase I study of navitoclax in patients with relapsed or refractory disease," Journal of Clinical Oncology, vol. 30, no. 5, pp. 488-496, 2012.

[30] L. Gandhi, D. R. Camidge, M. Ribeiro de Oliveira et al., "Phase I study of navitoclax (ABT-263), a novel Bcl-2 family inhibitor, in patients with small-cell lung cancer and other solid tumors," Journal of Clinical Oncology, vol. 29, no. 7, pp. 909-916, 2011. 
[31] A. J. Souers, J. D. Leverson, E. R. Boghaert et al., “ABT-199, a potent and selective BCL-2 inhibitor, achieves antitumor activity while sparing platelets," Nature Medicine, vol. 19, no. 2, pp. 202-208, 2013.

[32] J. Ren, G. Li, W. Zhao, L. Lin, and T. Ye, "Norcantharidin combined with ABT-737 for hepatocellular carcinoma: therapeutic effects and molecular mechanisms," World Journal of Gastroenterology, vol. 22, no. 15, pp. 3962-3968, 2016.

[33] R. Zheng, Z. You, J. Jia et al., "Curcumin enhances the antitumor effect of ABT-737 via activation of the ROS-ASK1-JNK pathway in hepatocellular carcinoma cells," Molecular Medicine Reports, vol. 13, no. 2, pp. 1570-1576, 2016.

[34] C.-M. J. Hu and L. Zhang, "Nanoparticle-based combination therapy toward overcoming drug resistance in cancer," Biochemical Pharmacology, vol. 83, no. 8, pp. 1104-1111, 2012.

[35] T. Lammers, V. Subr, K. Ulbrich et al., "Simultaneous delivery of doxorubicin and gemcitabine to tumors in vivo using prototypic polymeric drug carriers," Biomaterials, vol. 30, no. 20, pp. 3466-3475, 2009.

[36] F. Greco and M. J. Vincent, "Polymer-drug conjugates: current status and future trends," Frontiers in Bioscience, vol. 13, no. 13, pp. 2744-2756, 2008.

[37] J. Zhang, J. Hu, H. F. Chan, M. Skibba, G. Liang, and M. Chen, "iRGD decorated lipid-polymer hybrid nanoparticles for targeted co-delivery of doxorubicin and sorafenib to enhance anti-hepatocellular carcinoma efficacy," Nanomedicine: Nanotechnology, Biology and Medicine, vol. 12, no. 5, pp. 13031311, 2016.

[38] X. Zhao, Q. Chen, Y. Li, H. Tang, W. Liu, and X. Yang, "Doxorubicin and curcumin co-delivery by lipid nanoparticles for enhanced treatment of diethylnitrosamine-induced hepatocellular carcinoma in mice," European Journal of Pharmaceutics and Biopharmaceutics, vol. 93, pp. 27-36, 2015.

[39] K. Li, H. Liu, W. Gao et al., "Mulberry-like dual-drug complicated nanocarriers assembled with apogossypolone amphiphilic starch micelles and doxorubicin hyaluronic acid nanoparticles for tumor combination and targeted therapy," Biomaterials, vol. 39, pp. 131-144, 2015.

[40] S. Liu, H. Lu, A. R. Neurath, and S. Jiang, "Combination of candidate microbicides cellulose acetate 1,2-benzenedicarboxylate and UC781 has synergistic and complementary effects against human immunodeficiency virus type 1 infection," Antimicrobial Agents and Chemotherapy, vol. 49, no. 5, pp. 1830-1836, 2005.

[41] C. Chen, Y. C. Cheng, C. H. Yu, S. W. Chan, M. K. Cheung, and P. H. F. Yu, "In vitro cytotoxicity, hemolysis assay, and biodegradation behavior of biodegradable poly(3-hydroxybutyrate)-poly(ethylene glycol)-poly(3-hydroxybutyrate) nanoparticles as potential drug carriers," Journal of Biomedical Materials Research. Part A, vol. 87A, no. 2, pp. 290-298, 2008.

[42] L. Miao, S. Guo, C. M. Lin, Q. Liu, and L. Huang, "Nanoformulations for combination or cascade anticancer therapy," Advanced Drug Delivery Reviews, vol. 115, pp. 3-22, 2017.

[43] J. Fang, H. Nakamura, and H. Maeda, "The EPR effect: unique features of tumor blood vessels for drug delivery, factors involved, and limitations and augmentation of the effect," Advanced Drug Delivery Reviews, vol. 63, no. 3, pp. 136-151, 2011.

[44] F. Quaglia, L. Ostacolo, G. de Rosa et al., "Nanoscopic core-shell drug carriers made of amphiphilic triblock and star-diblock copolymers," International Journal of Pharmaceutics, vol. 324, no. 1, pp. 56-66, 2006.
[45] E. Jäger, A. Jäger, P. Chytil et al., "Combination chemotherapy using core-shell nanoparticles through the self-assembly of HPMA-based copolymers and degradable polyester," Journal of Controlled Release, vol. 165, no. 2, pp. 153-161, 2013.

[46] S. Lv, Z. Tang, M. Li et al., "Co-delivery of doxorubicin and paclitaxel by PEG-polypeptide nanovehicle for the treatment of non-small cell lung cancer," Biomaterials, vol. 35, no. 23, pp. 6118-6129, 2014.

[47] E. Fisicaro, C. Compari, and A. Braibanti, "Entropy/enthalpy compensation: hydrophobic effect, micelles and protein complexes," Physical Chemistry Chemical Physics, vol. 6, no. 16, pp. 4156-4166, 2004.

[48] E. Truzzi, C. Bongio, F. Sacchetti et al., "Self-assembled lipid nanoparticles for oral delivery of heparin-coated iron oxide nanoparticles for theranostic purposes," Molecules, vol. 22, no. 6, p. 963, 2017.

[49] B. Kang, O.-K. Kwon, H. Chung, W. Yu, and J. Kim, "Taxanes in the treatment of advanced gastric cancer," Molecules, vol. 21, no. 5, p. 651, 2016.

[50] Q. Hu, W. Sun, C. Wang, and Z. Gu, "Recent advances of cocktail chemotherapy by combination drug delivery systems," Advanced Drug Delivery Reviews, vol. 98, no. 1, pp. 19-34, 2016.

[51] J. G. Eley, V. D. Pujari, and J. McLane, "Poly (lactide-coglycolide) nanoparticles containing coumarin-6 for suppository delivery: in vitro release profile and in vivo tissue distribution," Drug Delivery, vol. 11, no. 4, pp. 255-261, 2008.

[52] I. Rivolta, A. Panariti, B. Lettiero et al., "Cellular uptake of coumarin-6 as a model drug loaded in solid lipid nanoparticles," Journal of Physiology and Pharmacology, vol. 62, no. 1, pp. 45-53, 2011.

[53] B. Xiao, M. K. Han, E. Viennois et al., "Hyaluronic acidfunctionalized polymeric nanoparticles for colon cancertargeted combination chemotherapy," Nanoscale, vol. 7, no. 42, pp. 17745-17755, 2015.

[54] E. Zhang, S. Luo, X. Tan, and C. Shi, "Mechanistic study of IR-780 dye as a potential tumor targeting and drug delivery agent," Biomaterials, vol. 35, no. 2, pp. 771-778, 2014.

[55] H. Cao, Y. Wang, X. He et al., "Codelivery of sorafenib and curcumin by directed self-assembled nanoparticles enhances therapeutic effect on hepatocellular carcinoma," Molecular Pharmaceutics, vol. 12, no. 3, pp. 922-931, 2015.

[56] C. E. Freter, T. C. Lee, M. E. Billingham, L. Chak, and M. R. Bristow, "Doxorubicin cardiac toxicity manifesting seven years after treatment. Case report and review," The American Journal of Medicine, vol. 80, no. 3, pp. 483-485, 1986. 


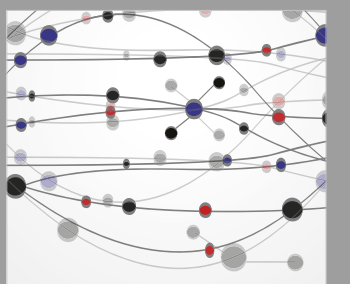

The Scientific World Journal
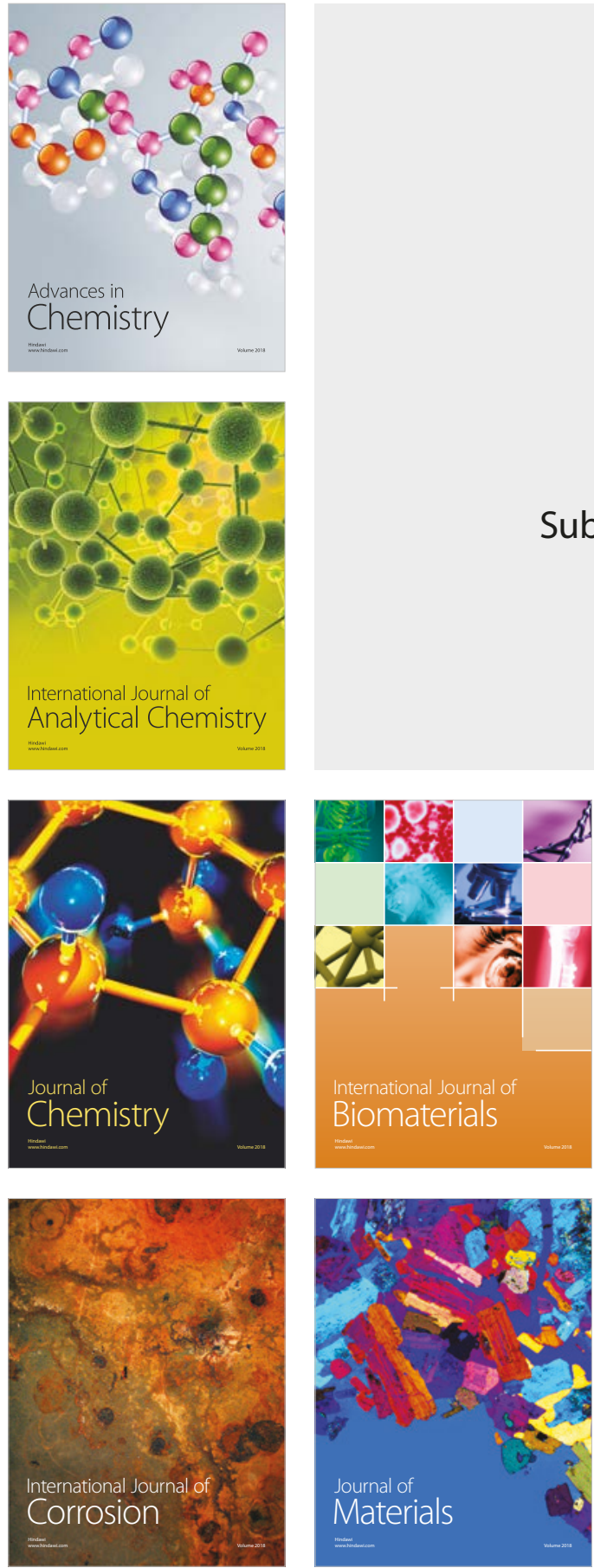

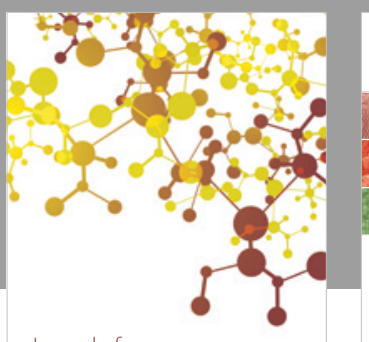

Journal of

Applied Chemistry
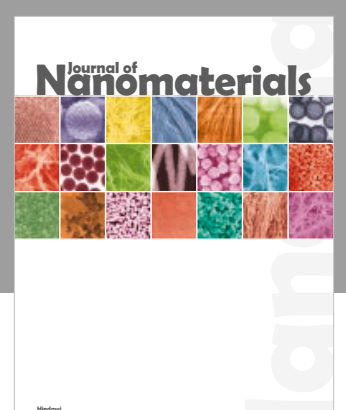

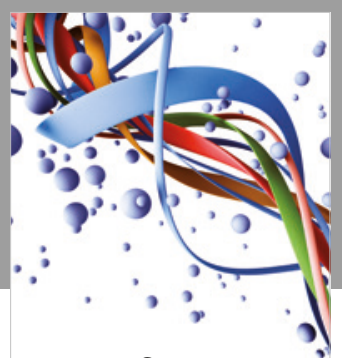

Scientifica

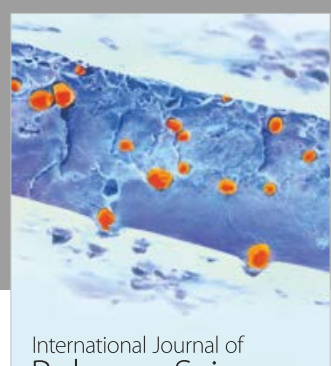

Polymer Science

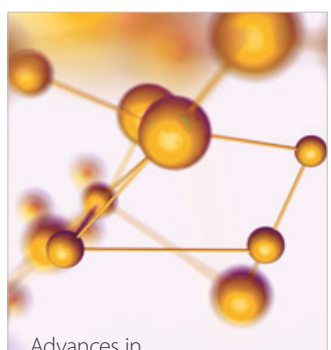

Physical Chemistry
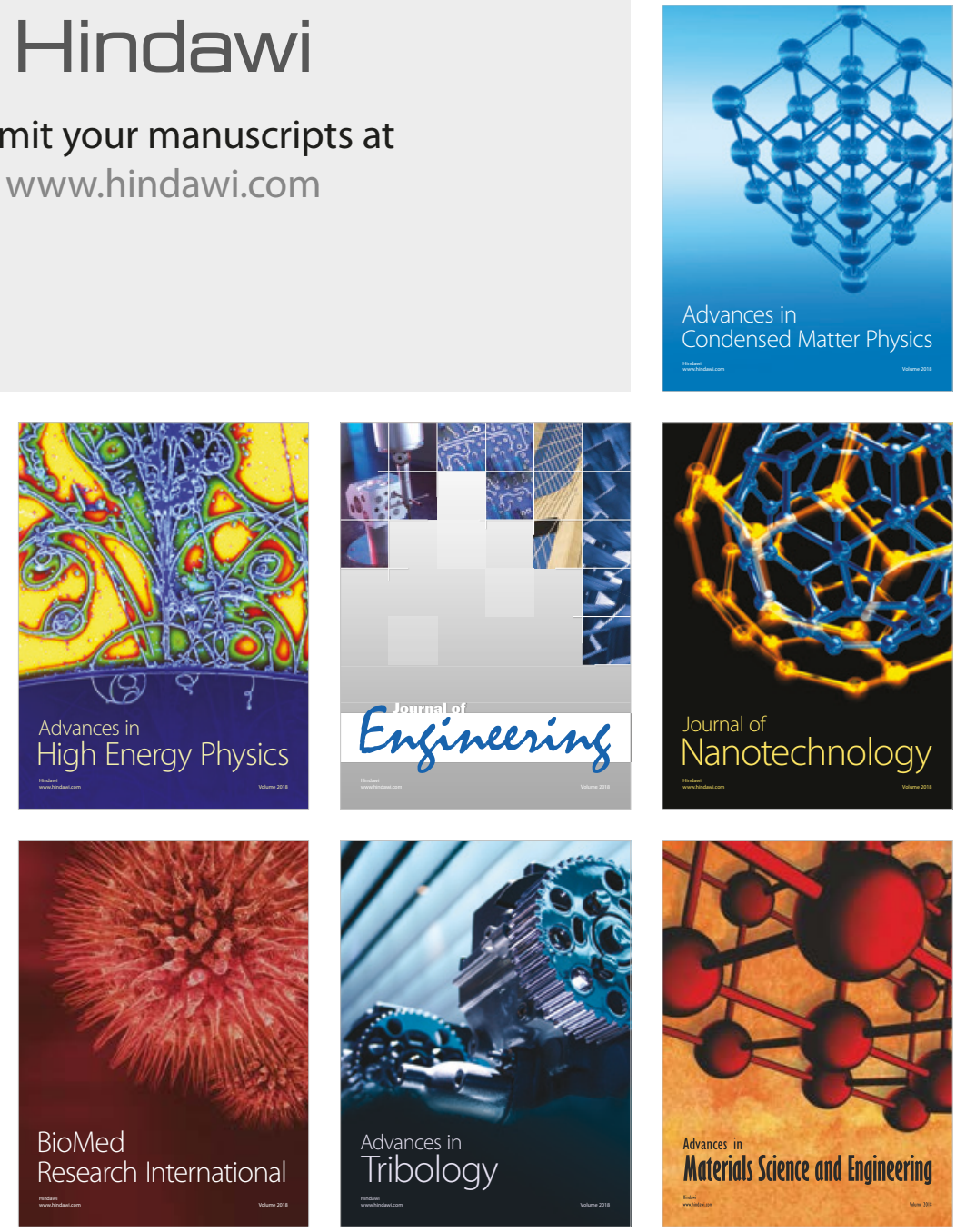